\title{
DE
}

BULGARIAN ACADEMY OF SCIENCES

CYBERNETICS AND INFORMATION TECHNOLOGIES • Volume 14, No 4

Sofia $\bullet 2014$

Print ISSN: 1311-9702; Online ISSN: 1314-4081

DOI: $10.1515 /$ cait-2014-0004

\section{Network Actual Traffic Prediction Algorithm Based on $\alpha$-stable Distribution and Wavelet Transformation}

\author{
Guobin Chen, Nanying Luo
}

RongZhi College, Chongqing Technology and Business University, Chongqing 400033, China Email: 759327776@qq.com

Abstract: In order to improve the prediction precision of wireless sensor network traffic, a new prediction algorithm (State Prediction algorithm based on $\alpha$-stable distribution $\alpha, S P-\alpha)$ is proposed, combined with $\alpha$-stable distribution and wavelet transformation. The algorithm proposed first defines the characteristics of $\alpha$-stable distribution and then gives the judge basis that obeys $\alpha$-stable distribution. At the same time, it reduces the prediction error of the actual traffic by fusion of the prediction results of $\alpha$-stable distribution with wavelet transformation. Finally, the paper thoroughly researches the key factors impacting on the new algorithm through simulations in OPNET and MATLAB. Compared with the performance of FARIMA model, the simulation results proved that $S P-\alpha$ algorithm has better adaptability.

Keywords: Wireless sensor network, prediction, $\alpha$-stable distribution, wavelet transformation, error.

\section{Introduction}

With the rapid development of wireless sensor networks, the performance of the actual traffic is increasingly considered by more and more people. The question whether the problem can be efficiently solved before a network congestion occurs, is often regarded. It plays a very important role in improving the network performance. 
Therefore, how to predict the next network traffic state has become the focus of the current study. At present the common methods of prediction are divided into linear and nonlinear. There are many linear methods, such as AR, ARMA model, etc. They have a relatively high prediction precision for the short traffic. However, the typically nonlinear prediction methods, such as FARIMA model and others, have a relatively high prediction precision for the long traffic. At the same time, with the depth of the study, other methods, such as wavelet transformation, chaotic model were introduced into the actual traffic prediction. The scholars at home and abroad have done a lot of researches on it. In [6] a variable scale method is presented which has super linear convergence of the improved ARMA prediction model and it has prediction of the smear method, based on the self correlation coefficient and partial correlation coefficient based on the actual traffic prediction. In [7] a based FARIMA model presents a prediction algorithm for probabilistic limitation, which aims at reducing the delay of the actual traffic. In [8], using the least squares support vector machine and fuzzy LSSVM training, an optimal sample subset of a fuzzy prediction algorithm is established to deeply study the actual traffic variability and the long cycle. Reference [9] discusses the problem that since the traditional prediction model is highly dependent on the training data, a prediction method of network weights of Back Propagation, based on wavelet technology and ant colony algorithm is developed to improve the prediction precision. In [10], based on the cosine function, the Logistic model is improved, using nonlinear time series analysis, method analysis and a Logistic model to describe the evolution trend of the actual traffic state and its chaotic characteristics. Using the wavelet transformation technology in [11], a prediction model of nonstationary characteristics is presented, that depicts the non-stationary characteristics of the actual traffic efficiently. The authors in [12] discuss the update prediction accuracy to establish an online fuzzy least square support vector machine method. But the effect of the time scales must be further considered.

On the basis of the above mentioned, this paper presents a new prediction algorithm for the actual traffic. It reduces the prediction error of the actual traffic by fusing $\alpha$-stable distribution [13-15] and the prediction results of wavelet transformation $[16,17]$. At the same time, it tests and proves the validity of the algorithm through mathematical simulations. The structure of the paper can be seen as follows: the first section gives $\alpha$-stable distribution, the second section presents the judgment basis of stable distribution and establishes the prediction algorithm, the third section carries out simulation experiments by using OPNET and MATLAB, the fourth section summarizes the paper.

\section{2. $\alpha$-stable distribution}

There are $M$ wireless nodes in the wireless sensor network shown in Fig. 1, which are divided into clusters. Each cluster has a cluster head node according to the residual energy and location, shown as a black circle in the picture. The cluster node is transmitted through the ordinary cluster head node data, and then all clusters gather into the base station for data exchange. In order to efficiently describe the 
performance of the wireless sensor networks traffic, we often use traditional methods, such as Poisson model, Gauss distribution, etc., and its research is based on the fact that the traffic has short correlation properties. However, with the development of the study, the results proved that the actual traffic shows self similarity and long correlation characteristics [18]. Thus, the following description model is proposed based on the Fractal Brown Motion model:

$$
A(t)=m t+\sqrt{a m} B^{H}(t),
$$

where $a$ is the variance coefficient, $m$ is the average arrival rate, $H$ is the self similar parameter, $B^{H}$ is the standard Fractal Brown Motion.

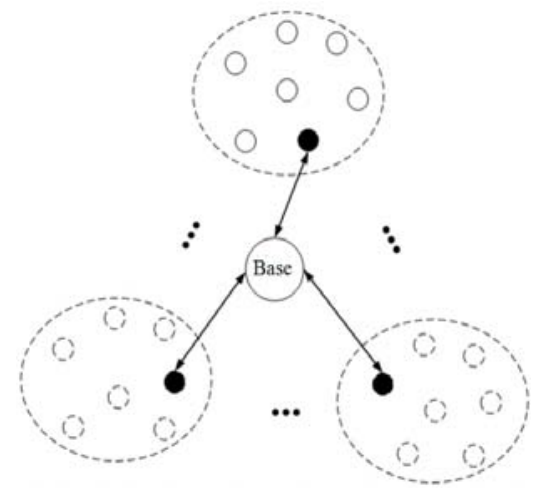

Fig. 1. Structure of a wireless sensor network

The research in recent years proved that the actual traffic has obvious scale characteristic and fractal characteristic, that is, it has self similarity and long correlation features on a large scale, but it has multi-fractal characteristics [19] on small scales. Therefore, according to the characteristic of the properties, such as wavelet transformation, chaotic model, FARIMA model are all applied for characterization of the traffic characteristics. But the chaos model, FARIMA model, is very complex for calculation, which cannot be efficiently put in practice. Although the wavelet transformation for multi scale characterization has many advantages, there is still a further optimization space for decomposition of the wavelet basis and scale.

Therefore, this paper combines with $\alpha$-stable distribution to describe the fractal characteristic and scale characteristic for the actual traffic characterization again. The wireless sensor network base station is used as a receiver, each wireless node is used as a data transmitting port, and the actual traffic arriving at the station is considered as random variable. Here we use $\alpha$-stable distribution to discuss its performance parameters. If the actual traffic complies with $\alpha$-stable distribution, it is recorded as $X \sim S_{\alpha}(\sigma, \beta, \mu)$, so that the characteristic function must meet the following:

$$
\Phi(\omega)=E\left[e^{j \omega X}\right]= \begin{cases}\exp \left\{j \mu \omega-|\sigma \omega|^{\alpha}\left[1-j \beta \operatorname{sgn}(\omega) \tan \left(\frac{\pi \alpha}{2}\right)\right]\right\}, & \alpha \neq 1, \\ \exp \left\{j \mu \omega-|\sigma \omega|^{\alpha}\left[1+j \beta \operatorname{sgn}(\omega) \frac{2}{\pi} \ln |\omega|\right]\right\}, & \alpha=1 .\end{cases}
$$


Here: $\alpha(0<\alpha<2)$ is the feature parameter, which is used to express the emergency degree and fractal dimension of the actual traffic; $\beta(-1 \leq \beta \leq 1)$ is the deviation parameter, which is used to indicate the deviation degree, $\alpha$ and $\beta$ can determine the shape of the density function of the data traffic distribution; $\sigma(\sigma \geq 0)$ is the deviation for the scale parameter; $\mu$ is the location parameter, which is used to express the deviation degree and the average distribution. If $\sigma=1$ and $\mu=0$ is the standard $\alpha$-stable distribution, $\operatorname{sgn}(\omega)$ is the sign function, expressed as:

$$
\operatorname{sgn}(\omega)=\left\{\begin{array}{cc}
1 & \text { if } \omega>1, \\
0 & \text { if } \omega=0 \\
-1 & \text { if } \omega<0
\end{array}\right.
$$

Combined with the stable distribution, it can define several characteristics of the network actual traffic.

Definition 1. If the actual traffic $X \sim S_{\alpha}(\sigma, \beta, \mu), 0<\alpha<2$, meets

$$
c x+b \sim S^{\alpha}(|c| \sigma, \operatorname{sgn}(c), c \mu+b) .
$$

Definition 2. If the actual traffic $X \sim S_{\alpha}(\sigma, \beta, \mu), 1<\alpha<2$, meets

$$
E(x)=\mu .
$$

Definition 3. If the actual traffic $X \sim S_{\alpha}(1, \beta, 0), 1<\alpha<2$, meets

$$
E\left(e^{-s X}\right)=\exp \left(-\frac{\sigma^{a} s^{\alpha}}{\cos (\pi \alpha / 2)}\right) \text {. }
$$

Definition 4. If the plurality actual traffic $X$ is an independent random variable, and its distribution meets $X_{i} \sim S_{\alpha}\left(\sigma_{i}, \beta_{i}, \mu_{i}\right) X_{i}$, then

$$
\left\{\begin{array}{l}
x_{1}+x_{2}+\cdots+x_{i} \sim S_{\beta, \sigma, \mu}^{\alpha}, \\
\sigma=\left(\sigma_{1}^{\alpha}+\sigma_{2}^{\alpha}+\cdots+\sigma_{i}^{\alpha}\right)^{1 / \alpha}, \\
\mu=\mu_{1}+\mu_{2}+\cdots+\mu_{i}, \\
\beta=\frac{\beta_{1} \sigma_{1}^{\alpha}+\beta_{2} \sigma_{2}^{\alpha}+\cdots+\beta_{i} \sigma_{i}^{\alpha}}{\sigma_{1}^{\alpha}+\sigma_{2}^{\alpha}+\cdots+\sigma_{i}^{\alpha}} .
\end{array}\right.
$$

The stability of the distribution means that a plurality of obedience to $\alpha$-stable distribution stacks with linear independent variables, the results also meet $\alpha$-stable distribution, so the traffic through polymerization still maintains $\alpha$-stable distribution characteristic.

Definition 5. If the actual traffic $X \sim S_{\alpha}(\sigma, \beta, \mu), 0<\alpha<2$, the variance meets:

$$
E|X|^{p}<\infty, \forall p, 0<p<\alpha .
$$

If $0<\alpha \leq 1$, the actual traffic $X$ is unlimited mean; If $1<\alpha<2$, the actual traffic $X$ is unlimited variance. The infinite variance attribute of the actual traffic can explain the sudden actual traffic. 
Definition 6. If the actual traffic is $X \sim S_{\alpha}(\sigma, \beta, \mu), 0<\alpha<2$, the tail probability distribution is

$$
\begin{aligned}
& P(X>x) \sim \sigma^{\alpha} \frac{1+\beta}{1} C_{\alpha} x^{-\alpha} . \\
& \text { Among them, } C_{\alpha}=\left\{\begin{array}{c}
\frac{1-\alpha}{\Gamma(2-\alpha) \cos (\pi \alpha / 2)} \text { if } \alpha \neq 1, \\
\frac{2}{\pi} \quad \text { if } \alpha=1 .
\end{array}\right.
\end{aligned}
$$

\section{State prediction algorithm}

The paper sets the ARM based DAS of the embedded gateway of a wireless sensor network, such as the environment, talking about the data transmission, including temperature and light sensation, from field sensor nodes to sink nodes, using a combined trigger mode based on timing and events. The sink nodes then pass in real time data through RS-232 serial port to the embedded network DAS, which receives data by multi-threading processing. After that, the real-time monitoring and data acquisition program will do the transmission to the server-side program, which will analyze and process the data and store them in the embedded database.

To check whether the actual traffic arriving meets $\alpha$-stable distribution, here are three specific test steps.

1. Assuming that the actual traffic meets $\alpha$-stable distribution, we can use the quartile method, maximum likelihood method, sample characteristic function method to estimate the parameters of the $\alpha$-stable distribution. In order to reduce the estimation error, we suggest an adopting maximum likelihood method, quartile method, sample characteristic function method, etc., to have comprehensive evaluation.

2. On the basis of getting estimated parameters of the $\alpha$-stable distribution, we can make the statistical probability distribution curve of the actual traffic and the probability density curve for the estimated parameters of $\alpha$-stable distribution. If the fitting degree of the two curves does not exceed the specified threshold, we can determine whether the actual traffic obeys $\alpha$-stable distribution, otherwise, we can consider that it does not obey $\alpha$-stable distribution.

3. For further verification of the above conclusion, the distribution characteristic of the Probability-Probability diagram and Quantile-Quantile diagram of the actual traffic analysis are combined. The basic principle of Quantile-Quantile is: a plurality of two groups of real traffic quantiles values were calculated separately and arranged in an ascending order. If the actual traffic distribution is near the angle of 45 degrees in a straight line, within the confidence intervals and located in $95 \%$ of the two groups, we can determine that the actual traffic of the two groups are from the same probability distribution. The Probability-Probability diagram can make up the inconvenience of Quantile-Quantile diagram for tail extreme observation. It judges the fitting degree of two groups by a probability density point of the actual traffic, its judge standard is whether the actual traffic in 
the Probability-Probability diagram is roughly distributed in a dip line for $45^{\circ}$ or so, and the deviation is in a certain range. If it is, the assumed distribution conforms to the actual traffic distribution.

If the actual traffic conforms to $\alpha$-stable distribution, we can predict the distribution through the characteristics of the traffic state to deal with the network congestion that may occur in advance.

Assuming that at time $t$, the actual traffic wireless sensor network base station $W_{t}$ receives $Z_{t}$, we mainly analyze the actual traffic for the time delay $D_{t}$ and the line length $L_{t}$, that is $Z_{t}=\left[D_{t}, L_{t}\right]$. To efficiently deal with the congestion phenomenon that may occur, we can analyze the actual traffic state through the present and past time to get the next moment traffic state $Z_{t+1}$, in order to implement a reasonable queue management.

\section{Algorithm}

Step 1. $W_{t}$ is the actual traffic which reaches the base station at time $t$. Firstly judge whether it meets the stable distribution by the method above mentioned, if it meets the requirement, then go to Step 2, otherwise go to Step 7.

Step 2. According to the calculation method given in [20], solve the time delay $D_{t+1}^{\prime}$ of the traffic and the line length $L_{t+1}^{\prime}$ at time $t+1$ :

$$
\left\{\begin{array}{l}
D_{t+1}^{\prime}=1-(1-\lambda) \sum_{n=0}^{\infty} \Omega_{k+n+1}((n+1) b-t), \\
L_{t+1}^{\prime}=(1-\lambda) \sum_{n=0}^{\infty} \Omega_{k+n}(n+1) b,
\end{array}\right.
$$

where $\lambda$ is the load size for the base station, $b$ is the buffer size, $\Omega_{k+n}$ is the distribution function of the actual traffic, the distribution function here is $\alpha$-stable distribution.

Step 3. At the same time, due to the sudden and scale characteristics of the actual traffic, the wavelet transformation of the traffic is introduced into an algorithm to process and predict the actual traffic, and fuse the results of prediction with the results obtained in Step 2 to reduce the error. According to the wavelet transformation formula, shown in the equations below, by using DB2 wavelet base to decompose the actual traffic for the time delay $D_{t}$ and the line length $L_{t}$ to obtain the scale coefficient of $j$ layer $a_{j}(k)$ and the wavelet coefficient $d_{j}(k)$ :

$$
\left\{\begin{array}{l}
\sqrt{2} \alpha_{j}(k)=\alpha_{j+1}(2 k)+\alpha_{j+1}(2 k+1), \\
\sqrt{2} d_{j}(k)=\alpha_{j+1}(2 k)-\alpha_{j+1}(2 k+1) .
\end{array}\right.
$$

Step 4. Using ARMA model to predict the wavelet coefficients, according to the estimation of $\operatorname{AR}(p)$ parameters $\tau(1), \tau(2), \ldots, \tau(p)$ and FIR filter, $A(z)=1+\sum_{k=1}^{p} \tau(k) z^{-k}$, output an approximate $\operatorname{MA}(q)$ process $\rho(1), \rho(2), \ldots, \rho(q)$ and obtain the parameters $p$ and $q$ at the same time. According to the equation given below can calculate the wavelet coefficient after prediction and use the inverse wavelet transformation after the synthesis prediction time delay $D_{t+1}^{\prime \prime}$ and captain $L$; 
the inverse wavelet, and the synthetic prediction after the delay in $D_{t+1}^{\prime \prime}$ and the line length $L_{t+1}^{\prime \prime}$ :

$$
\left\{\begin{array}{l}
D_{t+1}^{\prime \prime}=-\sum_{k=1}^{p} \tau_{k} D_{t-k}+\sum_{k=0}^{q} \rho_{k} u_{t-k}, \\
L_{t+1}^{\prime \prime}=-\sum_{k=1}^{p} \tau_{k} L_{t-k}+\sum_{k=0}^{q} \rho_{k} u_{t-k} .
\end{array}\right.
$$

Step 5. According to Steps 2 and 4 in order to obtain information about the time delay of the actual traffic at time $t+1$ and the line length, below is the fusion processing to reduce the prediction error,

$$
\left\{\begin{array}{c}
D_{t+1}=\phi D_{t+1}^{\prime}+\varphi D_{t+1}^{\prime \prime}, \\
L_{t+1}=\phi L_{t+1}^{\prime}+\varphi L_{t+1}^{\prime \prime},
\end{array}\right.
$$

where the fusion parameters $\phi$ and $\varphi$ indicate the weight distribution of the results for two predictions. It can be dynamically adjusted to obtain optimal results, and $0 \leq \phi \leq 1,0 \leq \varphi \leq 1, \phi+\varphi=1$.

Step 6. Assuming that $T=t+1$, go to Step 1 , repeatedly calculate the actual traffic vector for the next moment, until it ends.

Step 7. End of the algorithm.

\section{Mathematical simulation}

In order to prove the validity of SP- $\alpha$ algorithm, we have realized simulation experiments combined with OPNET and MATLAB. Firstly, the wireless sensor network topology structure, shown in Fig. 2 must be set up in OPNET. It totally produces ten clusters, each cluster has five nodes, and sets the link capacity to 4 Mbps, a delay of $20 \mathrm{~ms}$, the ordinary node buffer capacity is 80 packets, the base station buffer capacity is 600 packets, the packet size is $128 \mathrm{~B}$, the fusion parameters $\phi=\varphi=0.5$, the wavelet decomposition level is 10 . At the same time, in order to illustrate the precision of the prediction algorithm, it is compared to FARIMA [21] model which is also used for the description of fractal characteristic. Using two kinds of the algorithm in order to have continuous 30 times simulations in the experimental environment above mentioned, taking the average value, Figs 3 and 4 indicate that the two algorithms influence the prediction results for the delay and the line length of the actual traffic. It can be seen from Figs 3 and 4 that the proposed SP- $\alpha$ algorithm is close to the actual traffic. However, FARIMA model has some errors. The reason is that FARIMA model needs complex computation, in practice it often delays the dynamic transformation of the real state, and thus is causing a too large error. The comprehensive error analysis of the experimental results show that the error is $15.33 \%$ and $20.97 \%$ for SP- $\alpha$ algorithm and FARIMA model respectively. 


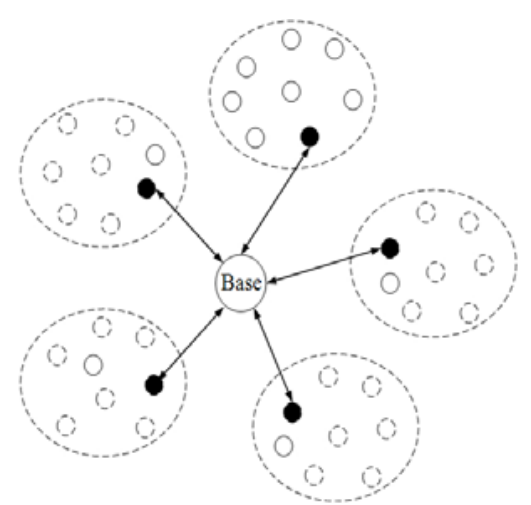

Fig. 2. Simulation environment

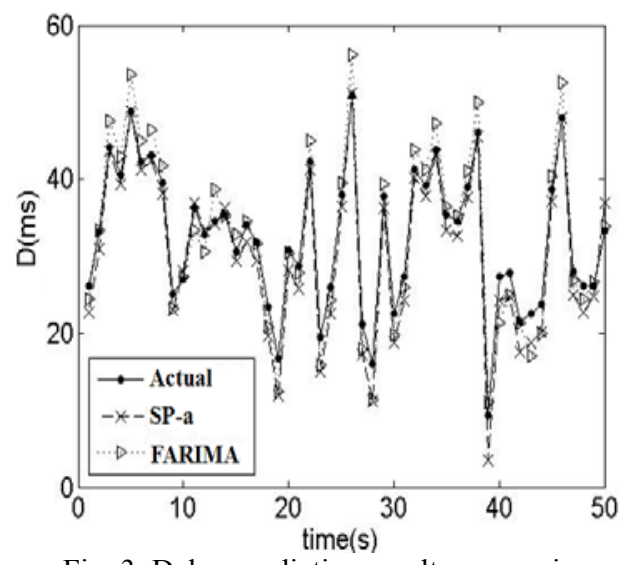

Fig. 3. Delay prediction results comparison

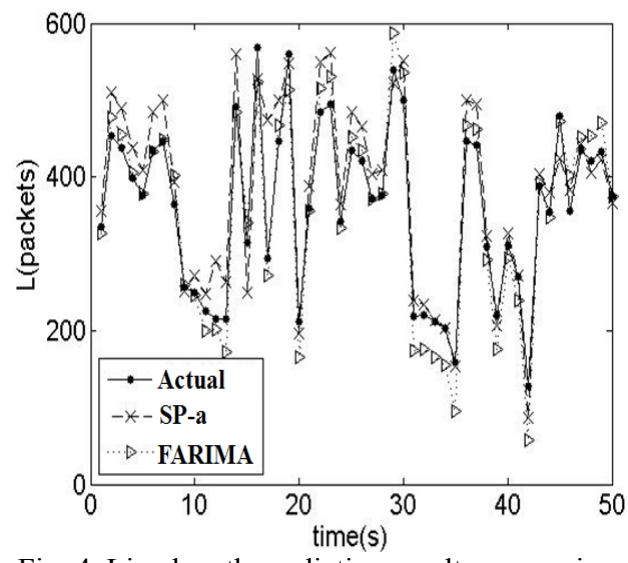

Fig. 4. Line length prediction results comparison

Secondly, the performance of SP- $\alpha$ algorithm is considered to discuss the effect on the performance of the changes in key parameters. For the algorithm performance under a different sudden state, Figs 5 and 6 show the changes that appear between the prediction errors of delay, the line length and the fusion parameter $\phi$. It can be seen from Fig. 5, that with the increase of $\delta$, the prediction errors of delay have a tendency from a decrease to an increase. The smaller parameter $\delta$ is, the bigger $\alpha$ is, the smaller the delay prediction error is. When the parameter $\delta$ is larger, $\alpha$ is bigger, the delay prediction error is bigger as well. Since $\alpha$ is larger, the sudden actual traffic is larger. SP- $\alpha$ algorithm has prediction, combined with $\alpha$-stable distribution and wavelet transformation. The smaller the parameter $\delta$ is, the greater the wavelet transformation influence is $\delta+\eta=1$. The wavelet transformation has a good effect on the elimination sudden state, thus the corresponding error is smaller. When the parameter is larger, $\alpha$-stable distribution plays an important role; it has a good effect in the treatment of a low sudden state. Therefore, the smaller $\alpha$ is, the smaller the prediction error is. A similar phenomenon can also be found in Fig. 6 . When the parameter is smaller, $\alpha$ is larger, the cohort prediction error is also larger. When the parameter is larger, it will jump. 


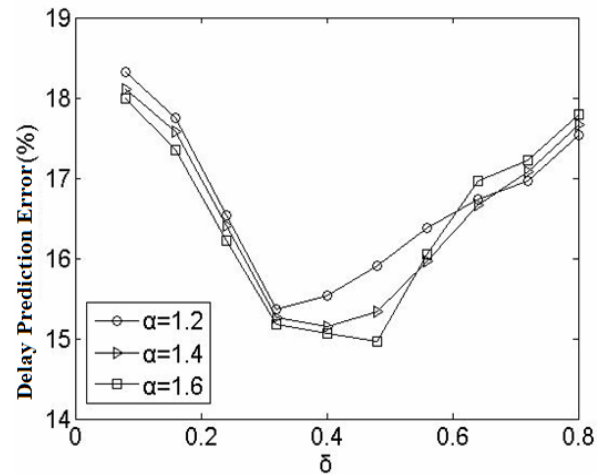

Fig. 5. Changes in the relationship between the delay prediction error and parameter $\delta$

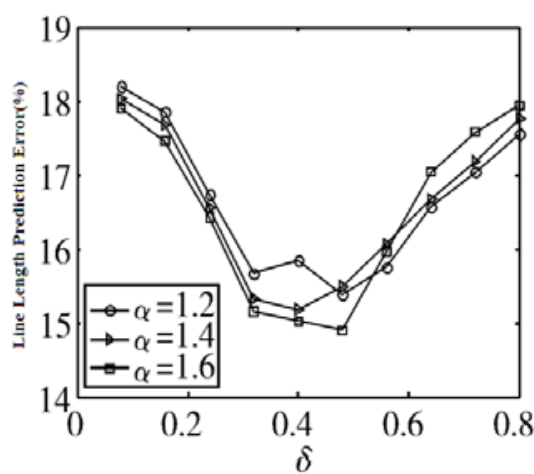

Fig. 6. Changes in the relationship between the line length prediction error and parameter $\delta$

At the same time, Figs 7 and 8 show the changes between the delay, line length and buffer $b$ under a different parameter $\alpha$. It can be seen from Fig. 7, that with the increase of buffer, the curve shows a progress increase trend. It conforms to the common phenomenon. The larger the buffer is, the more packets can be accommodated, thereby causing the transformed packet queuing time increase. In a small buffer, the bigger $\alpha$ is, the longer the delay is, while in the large buffer, the bigger $\alpha$ is, the shorter the delay is, which usually has different comprehension. A similar phenomenon is also shown in Fig. 8. In a small buffer, the larger $\alpha$ is, the smaller the line length is. In a large buffer, the larger $\alpha$ is, the larger the line length is. The reason for this phenomenon is mainly affected by limited buffers effect of truncation; when the buffer is in full state, it will directly discard subsequent data packets, which affects the long-range correlation of the traffic.

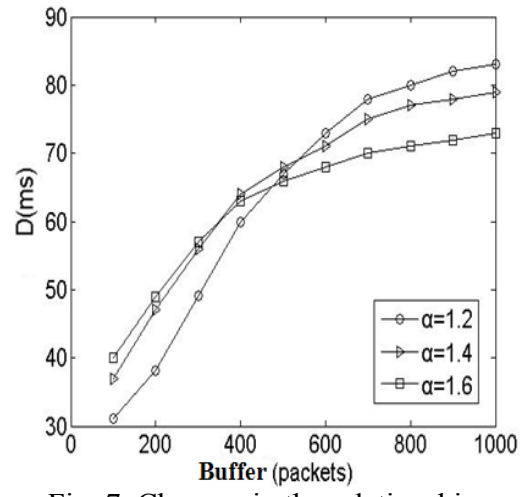

Fig. 7. Changes in the relationship between delay and buffer

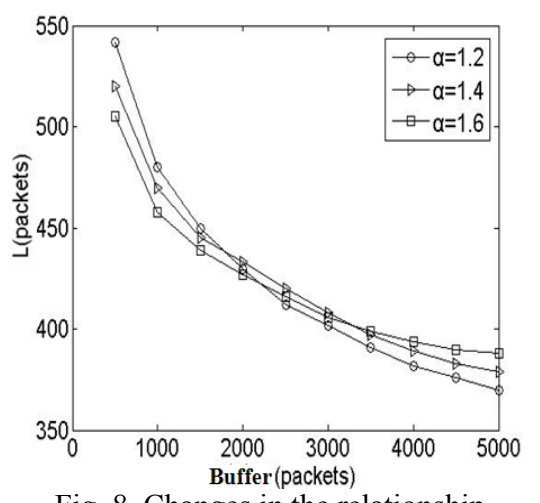

Fig. 8. Changes in the relationship between Line length and buffer

In the later network executing phase, some improvements must be made in the network management and implementation aspects as follows:

1. Realize the node monitoring, mobile node controlling, maintenance of the sink node linked routes, and the sensor node's function of tracing neighbor nodes. 
2. Provide network maintenance and diagnose services. Give the users access authorization to the network management service, including the functions of data acquisition, processing, analysis, and fault handling. This requires a new fully distributed network management mechanism according to the energy-constrained, self-organizing, and node-accidental-damage features of the sensor network.

3 . To ensure the network connection and efficient data transmission, save energy, and prolong the duty cycle, some nodes will be put into hibernation according to certain regulations. In this context, the topology management aims to coordinate all the nodes in the sensor network, and design an algorithm that ensures the network connection and the demand-driven network connection.

4. To prolong the lifetime of a network, energy must be used reasonably and efficiently. Because of the main tasks of sensor nodes, which are data acquisition, processing and transmission, the energy consumption concentrates mainly on two parts: computing consumption and communication consumption. The former is consumed in data collection and preprocessing, the latter in data communication between nodes. Considering these two factors, proper solutions must be made to realize an efficient energy management system.

\section{Conclusion}

For the prediction precision of the actual traffic in a wireless sensor network, a new prediction SP- $\alpha$ algorithm is proposed. At first it defines the characteristics of $\alpha$-stable distribution, and then it gives the judge basis that the actual traffic obeys its distribution. At the same time, it sets up an actual traffic prediction method combined with $\alpha$-stable distribution and wavelet transformation. It aims at reducing the prediction error of the actual traffic by fusing the prediction results of $\alpha$-stable distribution and wavelet transformation. Finally, the paper thoroughly researches the key factors impacting on the new algorithm through simulating OPNET with the help of MATLAB. Compared with the performance of FARIMA model, the simulation results proved that SP- $\alpha$ algorithm has better adaptability. In a subsequent study it is considered to establish a set of wireless sensor network prediction and an evaluation model that combines with the chaotic model and other prediction methods.

Acknowledgements: This work was supported by the Research Foundation of Chongqing Municipal Education Commission Science and Technology under Grant No KJ133103; Natural Science Foundation of Jiangsu Province under Grant No BK2011152; National Key Laboratory for Computer Science, subject to the Chinese Academy of Sciences under Grant No CSYSKF0908.

\section{References}

1. Li i o, H., C. Lee, S. P. Ke di a. Mobile Anchor Positioning for Wireless Sensor Networks.IET Communications, Vol. 5, 2011, No 7, 914-921.

2. Y i c k, J., B. M u k h e r j e e, D. G h o s a ld. Wireless Sensor Network Survey. - IEEE Computer Networks, Vol. 52, 2008, No 12, 2292-2330. 
3. W u, X. B., G. C h e n, S. K. D a s. Avoiding Energy Holes in Wireless Sensor Networks with Non Uniform Node Distribution. - IEEE Transactions on Parallel and Distributed Systems, Vol. 19, 2008, No 5,710-720.

4. Ch a n g, Y. C., Z. S. L in, J. L. Ch e n. Cluster Based Self Organization Management Protocols for Wireless Sensor Networks. - IEEE Transactions on Consumer Electronics, Vol. 52, 2006, No $1,75-80$

5. Lu o, X., M. D on g, Y. Hu a ng. On Distributed Fault Tolerant Detection in Wireless Sensor Networks. - IEEE Transactions on Computers, Vol. 55, 2006, No 1, 58-70.

6. W e i, S h a n, H e Q u n. Research of the Optimizing and Testing of Forecasting Model Based on the Non-Linear Time Series. - Chinese Journal of Electronics, Vol. 36, 2008, No 12, 2485 2489.

7. Yang, Song, Tu Xia omin, Fei Minrui. Internet Time-Delay Prediction Based on FARIMA Model. - Chinese Journal Scientific Instrument, Vol. 33, 2012, No 4, 757-763.

8. We n, Xiangxi, Meng Xiangru, Ma Zhiqiang, Zhang Yong chun. The Chaotic Analysis and Trend Prediction on Small-Time Scale Network Traffic.- Chinese Journal of Electronics, Vol. 40, 2012, No 8, 1609-1616.

9. Li, D and an, Z hang Runt ong, W a n g Chu an che n, X i a o D ong po. A New Network Traffic Prediction Model Based on Ant Colony Algorithm in Cognitive Networks. - Chinese Journal of Electronics, Vol. 39, 2011, No 10, 2245-2250.

10. Li, Chao, Zhao Hai, Zhang Jun. Network Delay Prediction Model Based on Chaotic Characteristic. - Chinese Journal of Electronics, Vol. 37, 2009, No 12, 2657-2661.

11. Xu, Ke, Xu Jinwu, B an Xia ojuan. Forecasting of Some Non-Stationary Time Series Based on Wavelet Decomposition. - Chinese Journal of Electronics, Vol. 29, 2001, No 4, 566-568.

12. Ye, Meiying, Wang Xi a odong, Zhang Haoran. Chaotic Time Series Prediction Based on Online Least Squares Support Vector Machine Regression. - Chinese Journal of Physics, Vol. 54, 2005, No 6, 2568-2573.

13. K a r a s a ri d is, A., D. H a t z i n a k o s. Network Heavy Traffic Modeling Using $\alpha$-Stable SelfSimilar Processes. - IEEE Transaction Communication, Vol. 49, 2001, No 7, 1203-1214.

14. Li, Xutao, Wang Shouyong. BCGM Based Rao Detection in $\alpha$-Stable Noise. - Chinese Journal of Electronics, Vol. 39, 2011, No 9, 2014-2018.

15. Zhang, Jinfeng, Qiu Tianshuang, Tang Hong. Robustness Analysis of DLMP Algorithm Under $\alpha$ - Stable Noise Environments. - Chinese Journal of Electronics, Vol. 35, 2007, No 3, 550-519.

16. Long, Tujing, S un Zhengshun, Li Chunwen, Jiang Peigang, Liu Jinhua. A New Multi-Fractal Wavelet Model for Network Traffic. - Chinese Journal of Computers, Vol. 27, 2004, No 8, 1074-1081.

17. Cong, Suo, Han Liangxiu, Li u Y an, Ga o Chuanshan. The Multi-Fractal Model Based on the Discrete Wavelet Transformation. - Journal of Communication, Vol. 24, 2003, No 5, 43-48.

18. L e 1 a n d, W. E., W. W i 11 ing e r, M. S. T a q q u, D. W i 1 s o n. On the Self-Similar Nature of Ethernet Traffic. - IEEE/ACM Transaction Networking, Vol. 2, 1994, No 1, 1-15.

19. Zhang, Junwen, Chen Haiwen, Chen Changjia. Study on Multi-Fractal Essential Cause of Internet Traffic. - Journal of Software, Vol. 13, 2002, No 3, 470-474.

20. Zhou, Manzhen, Quan Jichuan, Guo Yan, Liu Xiaoming, Su Zhaolong. Performance Analysis of Self-Similar Traffic Based on Bulk Queue. - Journal of System Simulation, Vol. 16, 2004, No 2, 306-309.

21. S a d e k, N., A. K hotanzad, T. Chen. ATM Dynamic Band width Allocation Using FARIMA Prediction Model. - In: Proceedings of International Conference on Computer Communications and Networks, 2003, No 2, 359-363. 